\title{
Papers
}

\section{Danish type gelsolin related amyloidosis: 654G-T mutation is associated with a disease pathogenetically and clinically similar to that caused by the $654 \mathrm{G}-\mathrm{A}$ mutation (familial amyloidosis of the Finnish type)}

Carl Peter J Maury, Mikko Liljeström, Gudrun Boysen, Tom Törnroth, Albert de la Chapelle, Eeva-Liisa Nurmiaho-Lassila

\begin{abstract}
Background-Familial amyloidosis of the Finnish type (FAF, Finnish hereditary amyloidosis) is caused by a 654G-A mutation in the gelsolin gene on chromosome 9 resulting in the expression of mutant Asn-187 gelsolin which is abnormally proteolytically processed generating amyloidogenic fragments that polymerise into amyloid fibrils. We have recently shown that in a Danish and a Czech family with a clinical syndrome similar to FAF, including corneal lattice dystrophy, cranial neuropathy and skin changes, the disease is caused by another mutation at the same position, namely 654G-T predicting a Tyrfor-Asp substitution at 187 in secreted gelsolin.
\end{abstract}

Aim-To undertake a closer examination of the Danish subtype of FAF and report immunohistochemical and biochemical findings.

Department of Medicine, University of Helsinki,

Kasarmikatu 11-13, FIN-00130 Helsinki, Finland

C P J Maury

M Liljeström

T Törnroth

Department of Neurology, Hvidovre Hospital, Hvidovre, Denmark

G Boysen

Folkhälsan Institute of Genetics, Helsinki, Finland

A de la Chapelle

Department of General Microbiology, University of Helsinki

E-L Nurmiaho-Lassila

Correspondence to: Professor Maury.

email: peter.maury@huch.fi

Accepted for publication 6 August 1999
Results-Immunostaining of plasma gelsolin isolated from heterozygous FAF of the Danish subtype revealed a pattern similar to that found in FAF-Asn 187. The epitope characteristic of the amyloid forming region as revealed by an amyloid specific antibody, whereas the $\sim 50 \mathrm{kDa}$ fragments are devoid of it. Compared with the wild-type gelsolin peptide (Asp-187), the corresponding mutant peptide (Tyr187) showed dramatically increased fibrillogenicity as revealed by quantitative thioflavine-T based fluorimetry; ultrastructurally, amyloid-like fibrils were formed by the mutant peptide. Immunohistochemistry showed that antibodies directed against residues 231-242 of secreted gelsolin, representing the carboxy terminus of the sequence forming the amyloid protein (residues 173-243) laid down in the tissues in a fibrillar form in FAF, specifically labelled the amyloid deposited in rectum and skin in the Danish (654G-T) subtype. $>60 \mathrm{kDa}$ gelsolin species contain an
Conclusions-The 654G-T mutation in the gelsolin gene gives rise to an amyloid disease clinically and pathogenetically similar to that caused by the 654G-A mutation. (F Clin Pathol 2000;53:95-99)

Keywords: amyloidosis; Finnish familial amyloidosis; gelsolin mutation 654G-T; fibrillogenesis

Familial amyloidosis of the Finnish type (FAF, Finnish hereditary amyloidosis, amyloid polyneuropathy type IV) is a dominantly inherited systemic amyloid disease characterised by cranial neuropathy, corneal lattice dystrophy, skin changes, and renal and cardiac manifestations. ${ }^{1-3}$ The disease is caused by a point mutation, $654 \mathrm{G}-\mathrm{A}$, in the gelsolin gene $\mathrm{e}^{4-6}$ resulting in the expression of amyloidogenic Asn-187 gelsolin and the accumulation of a 71 residue fragment of mutant gelsolin, Ala173Met243, as amyloid fibrils in the tissues. ${ }^{78}$ The Asn-187 gelsolin mutation has been found in all FAF patients so far described from Finland, as well as in occasional American, Dutch, and Japanese families. ${ }^{9-14}$ In contrast, in a Danish family and a Czech family with clinical syndromes similar to FAF, we found another mutation, namely $654 \mathrm{G}-\mathrm{T}$, in the gelsolin gene predicting a Tyr for Asp mutation at position 187 in secreted gelsolin. ${ }^{10}$ We have now undertaken a closer examination of the Danish subtype of FAF and report the immunohistochemical findings and the detection of an abnormal fragmentation of mutant gelsolin in plasma. We furthermore show that the substitution of Asp with $\mathrm{Tyr}$ at position 187 in synthetic gelsolin peptides is associated with markedly accelerated fibrillogenesis.

\section{Methods}

TISSUE AND PLASMA SAMPLES

We studied biopsy specimens from skin and rectum from three siblings of a Danish family with familial amyloidosis with slowly progressive cranial neuropathy and corneal lattice dystrophy. ${ }^{15}$ The $654 \mathrm{G}-\mathrm{T}$ mutation in gelsolin in this family was identified by allele specific 
Gelsolin

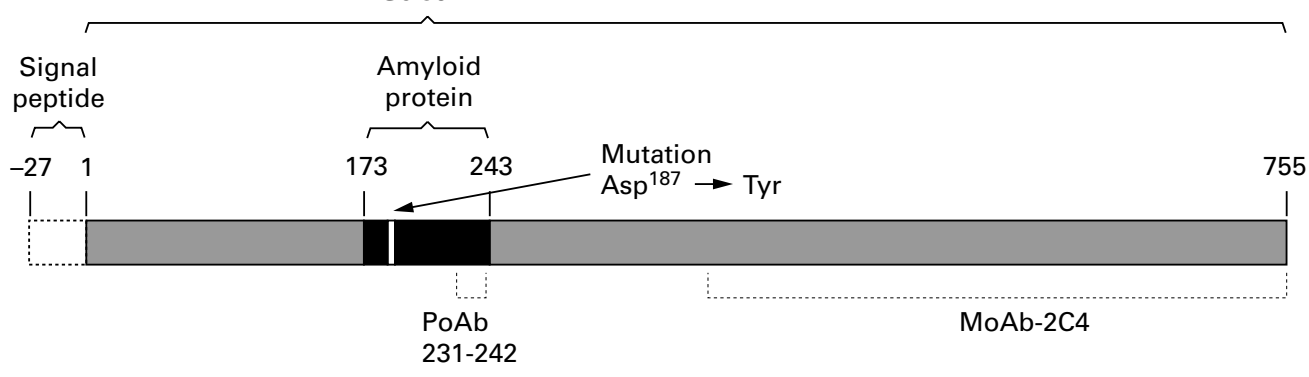

Figure 1 Schematic presentation of the amyloid forming region (residues 173-243) and mutation site in secreted gelsolin (residues 1-755) in Danish-type FAF, as well as the regions of the gelsolin molecule to which the amyloid specific polyclonal antibody (PoAb 231-242) and the monoclonal antibody MoAb-2C4 are directed.

oligonucleotide slot-dot hybridisation and by sequencing. ${ }^{10}$ Plasma gelsolin was isolated from a 57 year old male patient of the same Danish family. He had suffered from cranial amyloidotic polyneuropathy for about 10 years at the time of sampling. As controls we used plasma gelsolin isolated from Finnish subjects with FAF (two heterozygous (male) and one homozygous (female) patients with an identified 654G-A gelsolin mutation), plasma from healthy controls, and tissue samples from patients with FAF (Asn-187; three cases), AA amyloidosis (two cases), and $\mathrm{AL}$ amyloidosis (one $\kappa$ and one $\lambda$ case).

\section{ANTIBODIES}

We used two different antigelsolin antisera (fig 1): murine monoclonal antigelsolin antibody (MoAb-2C4) raised using a chymotryptic $47 \mathrm{kDa}$ C-terminal actin binding fragment of gelsolin (Sigma), and polyclonal rabbit antiamyloid antibodies (PoAb231-242) raised using synthetic VHVSEEGTEPEA amide corresponding to the carboxy terminus (residues 231-242) of the region forming the amyloid protein in $\mathrm{FAF}^{7}{ }^{16}$ The polyclonal antibodies were purified by immunoaffinity chromatography on Sepharose coupled to a gelsolin peptide corresponding to residues 231-241 in secreted gelsolin.

IMMUNOBLOTTING

For western blots, proteins were separated on $10 \%$ SDS-PAGE, electroblotted on nitrocellulose membranes, and detected with murine monoclonal or rabbit polyclonal antibodies using as second antibodies peroxidase or alkaline phosphatase conjugated rabbit or swine immunoglobulins to murine or rabbit immunoglobulins (Daka), respectively. Development was performed with 3,3'-diaminobenzidine tetrahydrochloride and hydrogen peroxide or with nitroblue tetratzolium and 5-bromo-4-chloro3-indolyl phosphate in dimethylformamide (AP Conjugate Substrate kit, Bio-Rad), respectively. As molecular standards, we used prestained protein marker, broad range (New England Biolabs) and prestained SDS page standards, low range (Bio-Rad).

\section{AFFINITY CHROMATOGRAPHY}

Isolation of gelsolin was carried out by immunoaffinity chromatography on a MoAb-2C4Sepharose column using Prot On Kit 2 (Multi- ple Peptide Systems) in the presence of $1 \mathrm{mM}$ EGTA. Affinity chromatography on immobilised cibacron blue F3GA was performed in the presence of $1 \mathrm{mM}$ EGTA as described previously. ${ }^{17}$ For routine testing, $100 \mu \mathrm{l}$ of plasma was chromatographed on $1.0 \times 1.5 \mathrm{~cm}$ cibacron blue column.

\section{HISTOCHEMISTRY}

Tissue specimens were studied for the presence of amyloid by routine congo red staining and polarisation microscopy. Immunoperoxidase staining was carried out as previously described. ${ }^{16}$ For immunogold staining, tissue sections $1 \mu \mathrm{m}$ thick were deparaffinised and after three washings incubated with 5\% normal goat serum diluted in phosphate buffered saline (PBS), $\mathrm{pH} 7.2$, containing $0.1 \%$ bovine serum albumin and $0.1 \%$ sodium azide. The sections were then incubated with the purified rabbit PoAb 231-242 antibodies for $30 \mathrm{~min}-$ utes, followed by three washings with PBS. Gold labelled goat antirabbit IgG (Auro Probe LM GAR, Amersham) at 1:40 dilution was added and incubated for one hour, followed by three washings with water. The sections were developed by a silver enhancement kit (Inten SEM, Amersham). ${ }^{18}$

IN VITRO FIBRILLOGENESIS

For fibril formation, synthetic peptides homologous to wild-type (SFNNGDCFILD-amide; residues 182-192) or mutant (SFNNGYCFILDamide and SFNNGNCFILD-amide representing residues 182-192) gelsolin were used (Multiple Peptide Systems). The purity of the peptides was $>95 \%$ as judged by high pressure liquid chromatography on a Vydac C18 column (fig 2).

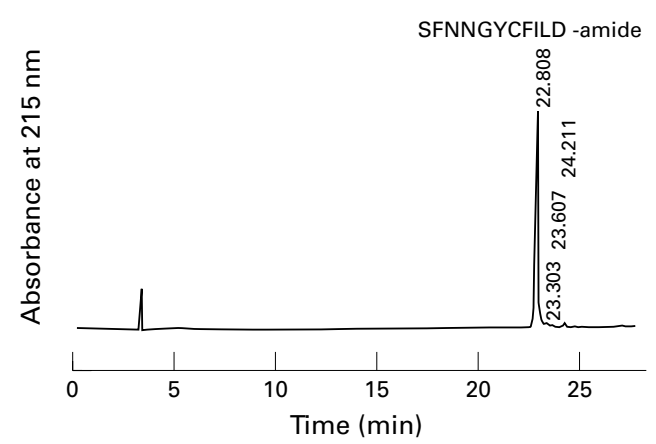

Figure 2 Reverse phase high pressure liquid chromatography on a Vydac C18 column of synthetic mutant 182-192 gelsolin peptide (Tyr-187). 


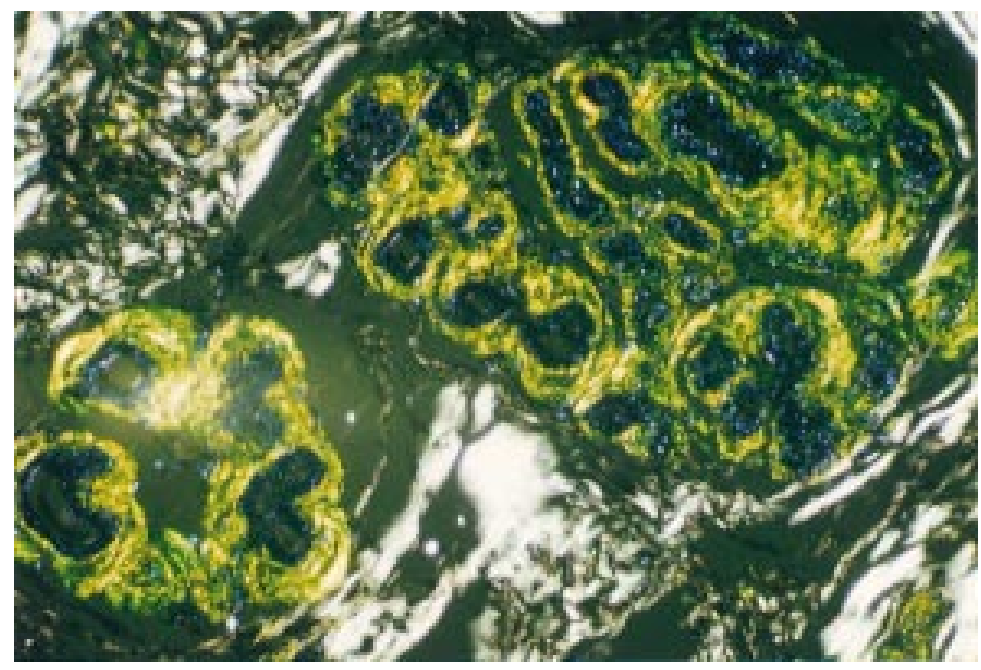

Figure 3 Dermal eccrine glands in Danish subtype (Tyr-187) familial amyloidosis showing prominent amyloid deposits in thickened basement membranes. Congo red staining, polarised light, magnification $\times 140$.

The peptides were dissolved in $10 \%$ acetic

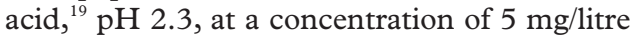
and analysed for Congo red positivity and green birefringence in polarised light after 15 minutes and overnight incubation at room temperature.

MONITORING OF FIBRIL FORMATION

Fibril formation was monitored by quantitative thioflavine-T fluorometry as described. ${ }^{2021}$ In this study we used the excitation and emission maxima of $450 \mathrm{~nm}$ and $482 \mathrm{~nm}$, respectively, and a Perkin-Elmer Luminescence LS 30 spectrometer.

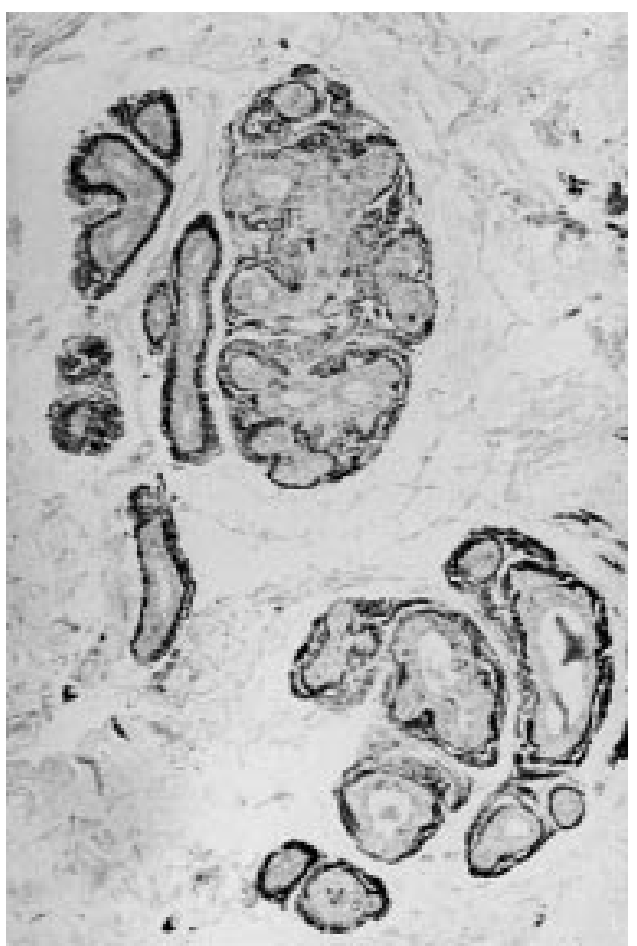

Figure 4 Immunogold-silver staining with amyloid specific antibody PoAb 231-242 of a skin biopsy in Danish subtype (Tyr-187) amyloidosis. Amyloid located in basement membranes is specifically stained. Magnification $\times 134$.

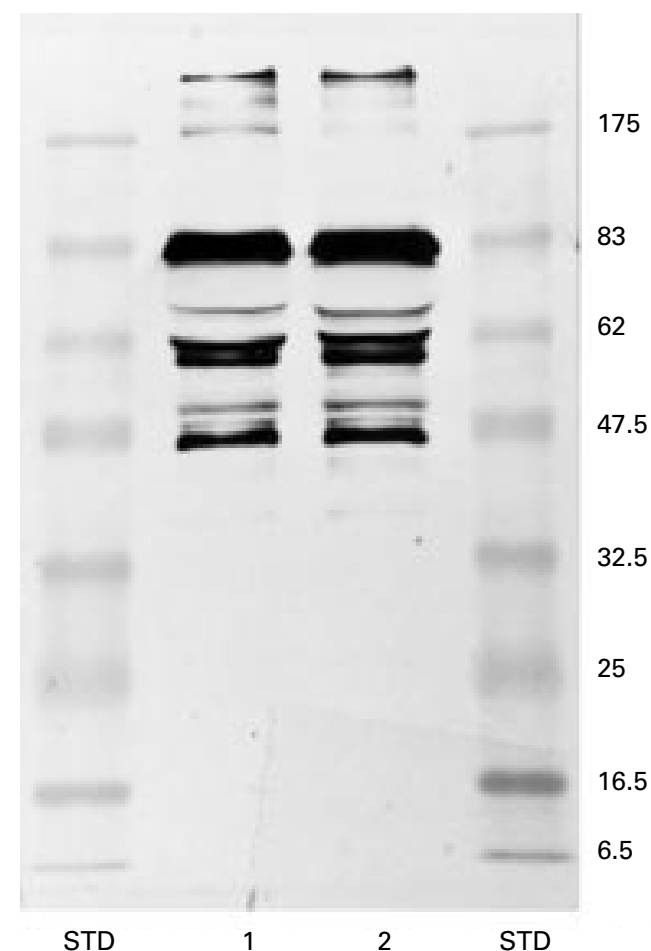

Figure 5 Comparison of heterozygous Danish subtype FAF (Tyr-187) and heterozygous Asn-187 FAF by immunoblotting by the monoconal MoAb-2C4 antibody. Lane 1, FAF Asn-187; lane 2, Danish FAF (Tyr-187); $S T D$, prestained molecular weight markers $(k D a)$.

\section{ELECTRON MICROSCOPY}

Negative staining electron microscopy was carried out on peptide samples that were dissolved in water or $10 \%$ acetic acid at a concentration of $5 \mathrm{mg} /$ litre, incubated at room temperature for 15 minutes and then kept frozen at $-20^{\circ} \mathrm{C}$. After thawing, the samples were diluted 1:1 in water, applied to a copper grid coated with pioloform and carbon, and stained with phosphotungstic acid adjusted to $\mathrm{pH} 6.5$ with potassium hydroxide. The grids were examined with a JEOL-JEM-100CX transmission electron microscope at an operating voltage of $60 \mathrm{kV}$.

\section{Results}

HISTOCHEMISTRY

Prominent amyloid deposits were found along the collagen fibres in rectal mucosa and the thickened basement membranes of the dermal eccrine glands (fig 3). The amyloid deposits were specifically stained by the antigelsolin antibody PoAb231-242 directed against the carboxy terminus of the region forming the amyloid subunit protein (figs 1 and 4). PoAb231-242 did not stain tissue amyloid in AA or AL amyloidosis.

\section{PLASMA GELSOLIN}

Plasma gelsolin from heterozygous Danish subtype FAF was isolated by affinity chromatography and subjected to immunoblotting with MoAb-2C4 antibody. In addition to normal sized gelsolin, lower molecular weight fragments were detected; the pattern in Danish heterozygous FAF was similar to that found in 


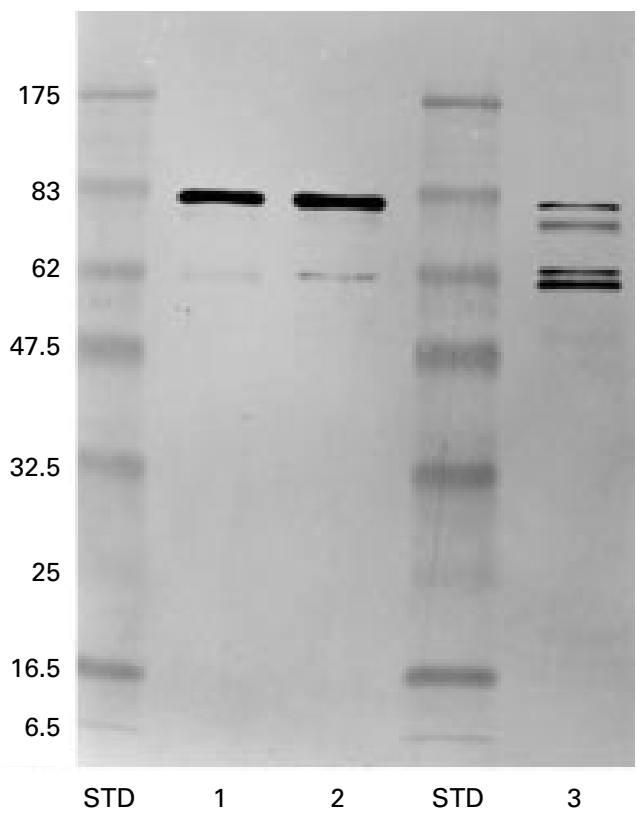

Figure 6 Immunostaining of the gelsolin fraction isolated from FAF plasma by affinity chromatography on an MoAb-2C4 Sepharose column by polyclonal antiamyloid antibodies directed against the $C$ terminus of the amyloid region (231-242). Lane 1, heterozygous FAF Tyr-187 (Danish); lane 2, heterozygous FAF Asn-187; lane 3, homozygous FAF Asn-187; STD, prestained molecular weight markers $(k D a$ ). Note that the $\sim 50 \mathrm{kDa}$ bands (fig 5) are not stained by the amyloid specific antibody as they are devoid of the amyloid forming sequence.

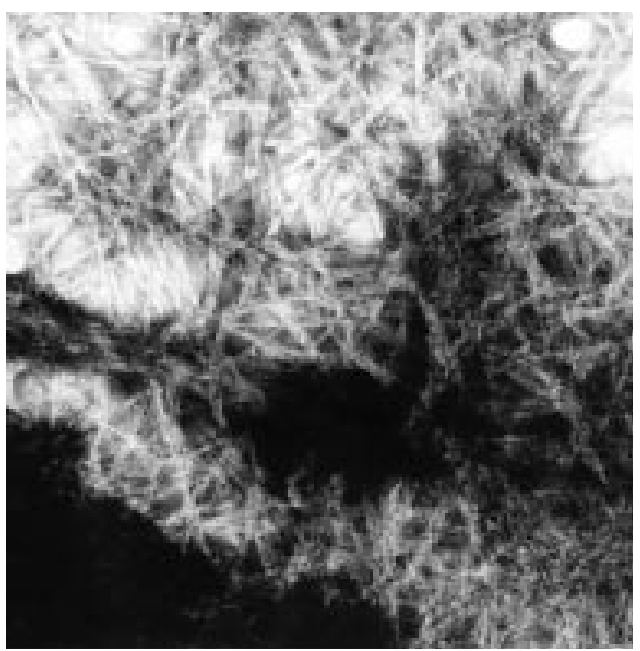

Figure 7 Electron micrograph of negatively stained protein assemblies formed by the mutant (Tyr-187) peptide, corresponding to residues 182-192 in gelsolin.

Magnification $\times 59595$.

heterozygous Finnish patients with the Asn187 mutation (fig 5). The antibody PoAb 231242, directed against the amyloid forming region of gelsolin (fig 1) recognised the $>60$ $\mathrm{kDa}$ gelsolin species, but not the $\sim 50 \mathrm{kDa}$ fragments (fig 6), which are devoid of the amyloid forming sequence.

IN VITRO AMYLOIDOGENESIS

Synthetic mutant Tyr-187 (Danish type) and Asn-187 (Finnish type) peptide, corresponding to residues 182-192 of gelsolin, formed amyloid-like fibrils in vitro (fig 7, Danish-type).

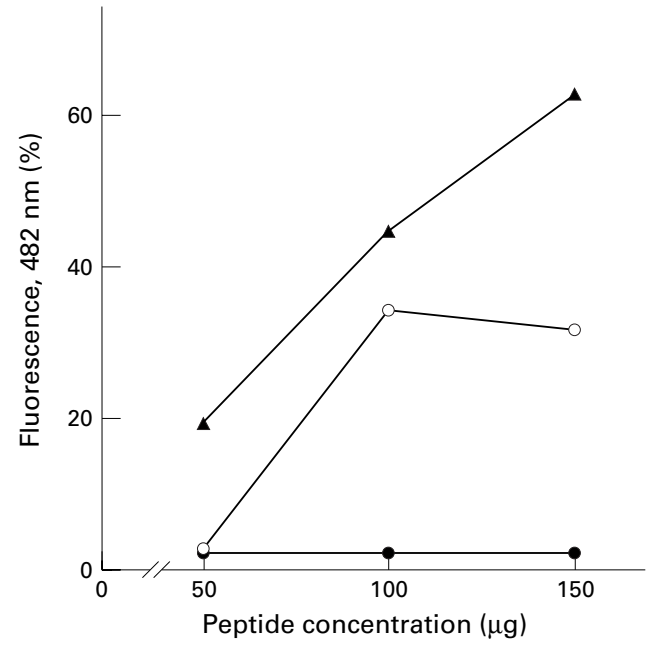

Figure 8 Comparison of amyloid fibril formation at different peptide concentrations by mutant (Tyr-187) gelsolin 182-192 peptide corresponding to Danish type amyloidosis (filled triangles), by mutant (Asn-187) gelsolin peptide 182-192 corresponding to Finnish amyloidosis (empty circles), and wild-type (Asp-187) gelsolin 182-192 peptide (filled circles), as measured by quantitative thioflavine-T based fluorimetry.

The mutant peptide aggregates were Congo red positive and showed green birefringence in polarized light. Fibril formation by the mutant peptides was dramatically enhanced as compared with the corresponding wild-type (Asp187) peptide (fig 8).

\section{Discussion}

The Danish type of familial amyloidosis with cranial neuropathy and corneal lattice dystrophy was described in 1979 by Boysen et al in five siblings of a family who had lived in Denmark for several generations. ${ }^{15}$ No genealogical connections to Finland could be shown for the family. The clinical features of the disease are very similar to those described by Meretoja in the Finnish families with hereditary amyloidosis, FAF. ${ }^{1}$ In contrast to FAF, where the gelsolin mutation has been identified as $654 \mathrm{G}-\mathrm{A},{ }^{4-6}$ a 654G-T mutation causes the Danish subtype of FAF. ${ }^{10}$ This mutation predicts a tyrosine for aspartic acid substitution at position 187 in secreted gelsolin.

The results of this study show that the biological consequences of the 654G-T mutation in gelsolin are very similar to those of the 654G-A mutation. Immunoblotting of plasma gelsolin in heterozygous Danish subtype FAF reveals a fragmentation pattern similar to that found in FAF (Asn-187) ${ }^{22}$ : in addition to normal sized gelsolin, a series of lower $\mathrm{Mr}$ C-terminal fragments is found. Thus the substitution of aspartic acid at position 187 in gelsolin with either asparagine or tyrosine is associated with abnormal degradation. The most likely explanation for the abnormal fragmentation, and the generation of the disease specific $65 \mathrm{kDa}$ fragment, ${ }^{22}{ }^{23}$ is that residue 187 represents a critical site where a substitution of an amino acid with a charged side chain (Asp) with one with an uncharged (Asn) or hydrophobic (Tyr) side chain induces a novel proteolysis site at $172-173$ by the mechanism of local unfolding. ${ }^{24}{ }^{25}$ This 
conclusion is also substantiated by cell culture studies that show that transfection of COS-1 cells ${ }^{26}$ with cDNA of Asp 187Asn/Tyr gelsolin is followed by the secretion in the medium of an abnormal fragment of about $68 \mathrm{kDa}$ size representing a C-terminal fragment of gelsolin, starting with Ala 173.

The experimental data also suggest that the gelsolin fragments containing the mutation site are highly fibrillogenic and rapidly polymerise into insoluble amyloid fibrils. Compared with the corresponding wild-type gelsolin peptide, mutant Tyr-187 peptide was very fibrillogenic as revealed by quantitative thioflavine- $T$ based fluorimetry. Moreover, electron microscopy showed that the fibrils created by the mutant Tyr-187 peptide were amyloid-like. Thus the 654G-T mutation appears to have a dual effect on amyloidogenesis: it is associated with the generation of a novel proteolysis site in gelsolin (172-173) resulting in the release of abnormal fragments that polymerise to amyloid fibrils during further proteolysis owing to the mutation induced, highly fibrillogenic amino acid sequence.

As shown previously for FAF(Asn-187), the circulating $\sim 65 \mathrm{kDa}$ gelsolin fragment, containing the amyloid sequence and derived from mutant gelsolin only, is FAF specific and can be used for diagnostic purposes. ${ }^{22}{ }^{23}$ The same test can be used for diagnosing the Danish subtype of FAF as the same sized fragment is generated in this disease. The disease specific fragment can be detected by the MoAb-2C4 antibody which recognises the non-amyloid region of the molecule (fig 1 ), or more specifically, by the amyloid directed PoAb 231-242 antibody (fig 1), as shown here.

In conclusion, the data presented here support the view that the $654 \mathrm{G}-\mathrm{T}$ mutation in the gelsolin gene gives rise to an amyloid disease clinically and pathogenetically similar to that caused by the $654 \mathrm{G}-\mathrm{A}$ mutation.

The study was supported by the Swedish Cultural Foundation and the Finska Läkaresällskapet, Finland.

1 Meretoja J. Familial systemic paramyloidosis with lattice dystrophy of the cornea, progressive cranial neuropathy, skin changes and various internal symptoms: a previously unrecognized heritable syndrome. Ann Clin Res 1969;1:314-24.

2 Maury CPJ, Kere J, Tolvanen R, et al. Homozygosity for the Asn 187 gelsolin mutation in Finnish-type familial Asn 187 gelsolin mutation in Finnish-type familial ics 1992;13:902-3.

3 Kiuru S, Matikainen E, Kupari M, et al. Autonomic nervous system and cardiac involvement in familial amyloidosis Finnish type (FAF). F Neurol Sci 1994;126:40-8.
4 Maury CPJ, Alli K, Baumann M. Finnish hereditary amyloidosis. Amino acid sequence homology between the amyloidosis. Amino acid sequence homology between the amyloid fibril protein

5 Maury CPJ, Kere J, Tolvanen R, et al. Finnish hereditary amyloidosis is caused by a single nucleotide substitution in the gelsolin gene. FEBS Lett 1990;276:75-7.

6 Levy E, Haltia M, Fernandez-Madrid I, et al. Mutation in gelsolin gene in Finnish hereditary amyloidosis. $\mathcal{F}$ Exp Med 1990;172:1865-7.

7 Maury CPJ, Baumann M. Isolation and characterization of cardiac amyloid in familial amyloid neuropathy type IV (Finnish): relation of the amyloid protein to variant gelsolin. Biochim Biophys Acta 1990;1096:84-6.

8 Maury CPJ. Gelsolin-related amyloidosis. Identification of the amyloid protein in Finnish hereditary amyloidosis as a

fragment of variant gelsolin. F Clin Invest 1991;87:1195-9. ila Chapelle A, Kere J, Sack GH, et al. Familial amyloidoFinnish families and an unrelated American family. Finnish families and an

10 de la Chapelle A, Tolvanen R, Boysen G, et al. Gelsolin derived familial amyloidosis caused by asparagine or tyrosine substitution for aspartic acid at residue 187. Nature Genet 1992;2:157-60

11 Paunio T, Sunada Y, Kiuru S, et al. Haplotype analysis in gelsolin-related amyloidosis reveals independent origin of dentical mutation (G654) of gelsolin in Finland and Japan. Hum Mutat 1995;6:60-5.

12 Paunio T. Molecular pathogenesis of familial amyloidosis, Finnish type. Helsinki: University of Helsinki, 1995. [Academic dissertation.]

13 Steiner RD, Paunio T, Uemichi T, et al. Asp187Asn mutation of gelsolin in an American kindred with familial amyloidosis, Finnish type (FAPIV). Hum Genet 1995;95:

Makishita H, Ikeda S-I, Yazaki M, et al. Postmortem pathological findings in a Japanese patient with familial amyloidosis, Finnish type (FAF). Amyloid Int $\mathcal{f}$ Exp Clin Invest 1996;3:134-9.

15 Boysen G, Galassi G, Kamieniecka Z, et al. Familial amyloidosis with cranial neuropathy and corneal lattice dystrophy. F Neurol Neurosurg Psychiatry 1979;42:1020-30.

16 Maury CPJ. Immunohistochemical localization of amyloid in Finnish hereditary amyloidosis with antibodies to gelsolin peptides. Lab Invest 1991;64:400-4.

17 Yamamoto H, Tarbayashi M, Egawa T, et al. Affinity separation of human plasma gelsolin on Affi-gel blue. $\mathcal{F}$ Biochem 1989;105:799-802.

18 Maury CPJ. Homozygous familial amyloidosis, Finnish type: demonstration of glomerular gelsolin-derived amyloid and non-amyloid tubular gelsolin. Clin Nephrol 1993;40:53-6.

19 Westermark P, Engström U, Johnson KH, et al. Islet amyloid polypeptide: pinpointing amino acid residues linked to amyloid fibril formation. Proc Natl Acad Sci USA 1990;87: 5036-40.

20 Naiki H, Higuchi K, Hosokawa M, et al. Fluorimetric determination of amyloid fibrils in vitro using the fluorescent dye thioflavine T. Anal Biochem 1989;177:244-9.

21 Maury CPJ, Nurmiaho-Lassila M-L. Creation of amyloid Maury CPJ, Nurmiaho-Lassila M-L. Creation of amyloid
fibrils from mutant ASN 187 gelsolin peptides. Biochem fibrils from mutant ASN 187 gelsolin
Biophys Res Commun 1992;183:227-31.

22 Maury CPJ, Rossi H. Demonstration of a circulating $65 \mathrm{~K}$ gelsolin variant specific for familial amyloidosis, Finnish type. Biochem Biophys Res Commun 1993;191:41-4.

23 Maury CPJ, Sletten K, Totty N, et al. Identification of the circulating amyloid precursor and other gelsolin metabolites in patients with G654A mutation in the gelsolin gene (Finnish familial amyloidosis): pathogenetic and diagnostic implications. Lab Invest 1997;77:299-304.

24 Maury CPJ, Nurmiaho-Lassila M-L, Rossi H. Amyloid fibril formation in gelsolin-derived amyloidosis. Definition of the amyloidogenic region and evidence of accelerated amyloid formation of mutant Asn-187 and Tyr-187 gelsolin peptides. Lab Invest 1994;70:558-64.

25 McLaughlin PJ, Gooch JT, Mannherz H-G, et al. Structure of gelsolin segment 1-actin complex and the mechanism of filament severing. Nature 1993;364:685-92.

26 Kangas H, Paunio T, Kalkkinen N, et al. In vitro expression analysis shows that the secretory form of gelsolin is the sole source of amyloid in gelsolin-related amyloidosis. Hum Mol Genet 1996;5:1237-43. 